\title{
Review and experiments on nuclear astrophysics
}

\author{
Christian Iliadis ${ }^{* \dagger}$ \\ Department of Physics and Astronomy, University of North at Carolina Chapel Hill, NC \\ 27599-3255, USA \\ E-mail: iliadis@unc.edu
}

\section{Art Champagne}

Department of Physics and Astronomy, University of North at Carolina Chapel Hill, NC 27599-3255, USA

E-mail: artc@physics.unc.edu

\begin{abstract}
We will present the basic fundamental ideas regarding nuclear processes occurring in stars. Starting from stellar observations, we will elaborate on some important quantum-mechanical phenomena governing nuclear reactions, will then continue with how nuclear reactions proceed in a hot stellar plasma, and then provide an overview of hydrostatic stellar burning stages. Furthermore, we will discuss direct measurements of nuclear reactions that are important for stellar burning.
\end{abstract}

Gran Sasso Summer Institute 2014 Hands-On Experimental Underground Physics at LNGS 22 September - 03 October 2014

INFN - Laboratori Nazionali del Gran Sasso, Assergi, Italy

\footnotetext{
* Speaker.

${ }^{\dagger}$ This work was supported by the U.S. Department of Energy under Contract No. DE-FG02-97ER41041.
} 


\section{Introduction}

The field of nuclear astrophysics started with basic questions regarding the Sun. Life on Earth depends on nuclear processes deep inside the Sun, but how exactly the nuclear transmutations occur was not understood for some time. The breakthroughs came at the end of the 1930's: Bethe and Critchfield [1] uncovered a sequential reaction chain fusing hydrogen $(\mathrm{H})$ to helium $(\mathrm{He})$, now referred to as the ppl chain, while Bethe [2] and von Weizsäcker [3] proposed a cyclic reaction sequence, now called the CNOI cycle, that has the same end result of synthesizing He from $\mathrm{H}$. For this early work, the Nobel prize was awarded to Hans Bethe in 1967. It is interesting to point out that Bethe originally thought that the Sun derives most of its energy via the CNO1 cycle. Part of the problem was that the key nuclear reaction cross sections were poorly known. When more reliable cross sections could be estimated in the 1950's, it became apparent that it is in fact the pp1 chain that governs the energy production in the Sun. The important lesson is that accurate nuclear physics information is crucial for our understanding of stars.

Some obvious questions followed immediately: how do other stars produce energy? How do they evolve and why do some of them explode? And perhaps the key question: where were the elements found on Earth produced? They were certainly not produced inside the Sun and, therefore, other processes are required to explain their origin. In this regard, the solar system abundance distribution of the nuclides became of paramount importance. A modern version is displayed in Fig. 1 and reveals a rather interesting structure. The different processes giving rise to the observed features were explained by Burbidge, Burbidge, Fowler and Hoyle [4] and by Cameron [5]. These papers laid the foundation of the modern theory of nuclear astrophysics. For this work, the Nobel prize was awarded to Willy Fowler in 1983.

Briefly, $\mathrm{H}$ and $\mathrm{He}$ are the most abundant elements and they were made in the Big Bang. The abundance curve then drops sharply by eight orders of magnitude. The species $\mathrm{Li}, \mathrm{Be}$, and $\mathrm{B}$ are so quickly destroyed inside stars that their production must take place elsewhere. In fact, they are believed to be produced by cosmic-ray spallation. The abundance curve increases sharply at $\mathrm{C}$ and $\mathrm{O}$. These are the most abundant elements after $\mathrm{H}$ and $\mathrm{He}$ and, incidentally, are the species life on Earth is based on. For increasing mass number the abundance curve decreases, but then produces a maximum near $\mathrm{Fe}, \mathrm{Co}$ and $\mathrm{Ni}$, referred to as the iron peak. Interestingly, these nuclides exhibit the largest binding energies per nucleon. So far, most of the species have been produced by nuclear reactions involving charged particles. To explain the origin of the nuclides located beyond the iron peak, however, fundamentally different processes are required. Those species are mainly produced via the capture of neutrons in the s-process and the r-process.

In this short introduction, we will focus on charged particle processes important for hydrostatic (as opposed to explosive) stellar nucleosynthesis and energy production in stars. It will become apparent how quantum-mechanical processes govern the evolution of large-scale objects in the Universe. This inter-connection is fascinating and remarkable, especially in view of the complex interplay of nuclear physics, hydrodynamics, atomic physics, and plasma physics in stars. We may state without exaggerating that after several decades of research, stellar evolution and nucleosynthesis are among the most successful complex theories humans possess. The account given here is based on a textbook [7], to which the student is referred for more detailed information.

The single most important stellar property that determines the evolutionary fate of a star is its 


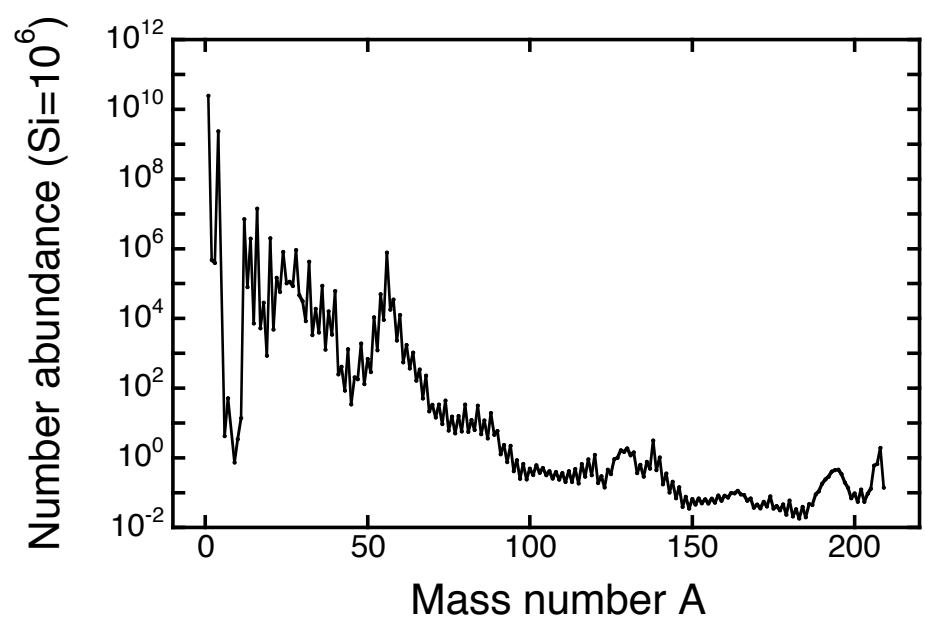

Figure 1: Abundances of the nuclides, normalized to the number of Si atoms, at the birth of the solar system. Data from Ref. [6].

mass. The larger the mass, the larger the temperature and pressure in the core. Thus nuclear energy must be generated at a faster pace in order to stabilize the star, implying a larger luminosity and a faster fuel consumption. Consequently, the larger the mass the shorter the stellar lifetime. Before continuing, it is worthwhile to discuss briefly some astrophysical phenomena.

We start with globular clusters. A particularly beautiful example is the cluster NGC 6752, shown in Fig. 2. Globular clusters are located in a spherical space surrounding the Galactic center, called the halo. A typical cluster consists of $10^{4}-10^{6}$ stars and is metal poor compared to the Sun, implying that it was formed during the early stages of Galactic evolution. The stars in a single cluster were formed around the same time from material of very similar composition. When plotting the luminosity versus surface temperature for many stars in a given globular cluster (Hertzsprung-Russell diagram), it is apparent that the stars occupy distinct regions in the diagram. This observation must then be explained by differences in their stellar mass. It is interesting that the age of a cluster can be determined by comparing the location of the turn-off point (i.e., the region in the HR diagram corresponding to those stars that have exhausted the $\mathrm{H}$ fuel in their core) with predictions from stellar evolution models, provided that accurate nuclear reaction cross sections are available. Such investigations yield ages for the oldest globular clusters of 12-13 Gy. This value represents an important lower limit for the age of the Universe, demonstrating how nuclear reaction cross sections are related to cosmological questions.

It is illuminating to describe briefly the evolution of low-mass stars $\left(M / M_{\odot}=0.4-2\right)$, including the Sun. At present the Sun converts H to He in the core via the pp1 chain (hydrogen burning). In about $5 \mathrm{~Gy}$, the $\mathrm{H}$ fuel in the core will be exhausted and the Sun will become a red giant star, fusing $\mathrm{H}$ to $\mathrm{He}$ via the $\mathrm{CNO} 1$ cycle in a shell surrounding an inert He core. The temperature in the core increases until $\mathrm{He}$ starts to fuse to $\mathrm{C}$ and $\mathrm{O}$ (helium burning), while $\mathrm{H}$ continues to burn in a shell surrounding the core. In this phase, the low-mass stars are referred to as horizontal branch stars. At some point, the $\mathrm{He}$ in the core is exhausted and the stars will burn He in a shell surrounding an inert $\mathrm{C}$ and $\mathrm{O}$ core, in addition to burning $\mathrm{H}$ to $\mathrm{He}$ in a shell surrounding the He burning region. 


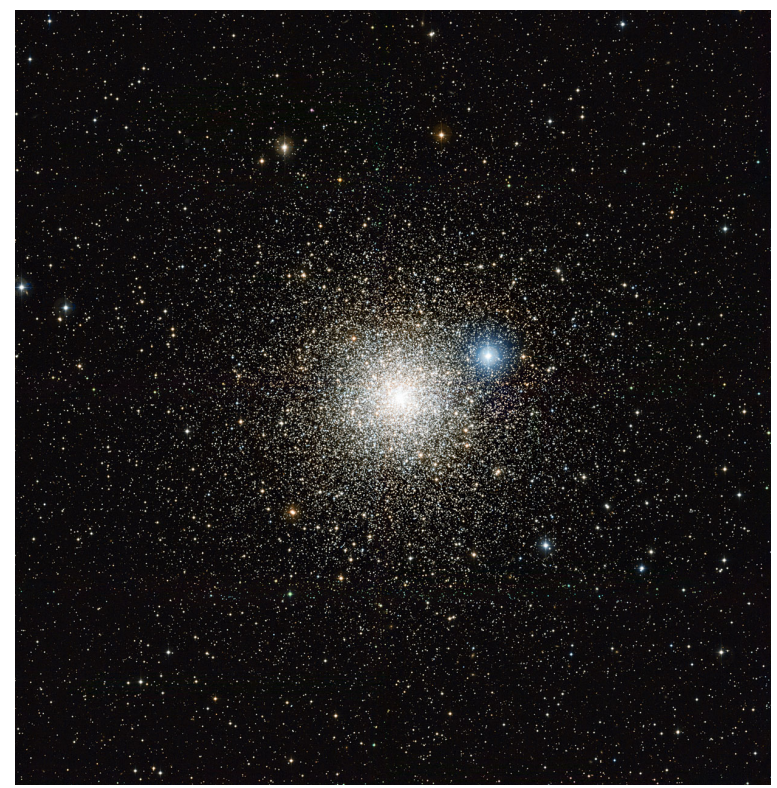

Figure 2: The globular cluster NGC 6752 in the southern constellation Pavo, located at a distance of about 13,000 light-years. It holds over 100 thousand stars, has a diameter of about 100 light-years, and is over 10 billion years old. It is also one of the brightest globular clusters in the night sky. Stars in this cluster, among them the ancient red giants (shown in yellow-orange hue), reveal remarkable elemental abundances in their atmospheres that are not yet understood [8]. Image credit: ESO.

This phase, which is referred to as the asymptotic giant branch (AGB), gives rise to thermal instabilities, where the $\mathrm{H}$ and $\mathrm{He}$ burning shells alternate as the main contributor to the luminosity. As a result, the star will experience a significant mass loss via a strong stellar wind. Later on, when the stellar surface becomes hot enough, the intense ultraviolet radiation ionizes the previously ejected matter, which begins to fluoresce brightly as a planetary nebula. Eventually, the $\mathrm{H}$ burning shell extinguishes and the low-mass star will end its existence as a white dwarf, consisting mainly of $\mathrm{C}$ and $\mathrm{O}$. It is supported by electron degeneracy pressure and cools slowly by radiating away its thermal energy. AGB stars are believed to be not only the main source of $\mathrm{C}$ and $\mathrm{N}$ in the Universe, but also of the main component of the s-process.

Intermediate-mass stars $\left(M / M_{\odot}=2-11\right)$ largely follow the same evolutionary sequence as their low-mass counterparts. One important difference is that the He core in intermediate-mass stars does not reach degeneracy and thus He burning proceeds quiescently. The more massive of these stars will survive beyond the AGB stage to ignite $\mathrm{C}$ in their cores under degenerate conditions. These stars also end their lives as white dwarfs, but with a composition of primarily $\mathrm{O}$ and $\mathrm{Ne}$, the major end-products of $\mathrm{C}$ burning.

Massive stars $\left(M / M_{\odot}>11\right)$ evolve very differently from low-mass stars. We will briefly describe the fate of a $25 M_{\odot}$ star of solar composition. After undergoing $\mathrm{H}$ and $\mathrm{He}$ burning, the core experiences further burning episodes. These are referred to as $\mathrm{C}-, \mathrm{Ne}-, \mathrm{O}-$, and $\mathrm{Si}$-burning (also called advanced burning stages), and will be explained in more detail below. For example, after the end of He burning the core consists of $\mathrm{C}$ and $\mathrm{O}$. The core contracts gravitationally, while raising the temperature and pressure, in order to stabilize the star. At some point, $\mathrm{C}$ begins to 
fuse, providing a source of nuclear energy and halting temporarily the contraction of the star. This cycle repeats while the core experiences more such burning stages. Furthermore, each time a given burning phase terminates in the core, it continues to burn in a shell surrounding the core. The duration of each subsequent nuclear burning phase decreases significantly. For example, while $\mathrm{H}$ burning may last many million years, Si burning may last only one day. The reasons are twofold. First, $\mathrm{H}$ burning releases far more energy per unit mass $\left(6 \times 10^{24} \mathrm{MeV} / \mathrm{g}\right)$ compared to $\mathrm{He}$ and $\mathrm{C}$ burning. Hence, the $\mathrm{H}$ fuel is consumed much more slowly. Second, the manner by which the star radiates energy (or "cools") changes dramatically. For $\mathrm{H}$ and He burning, the nuclear energy generated in the core eventually reaches the surface and is radiated as photons. Beyond He burning, starting with $\mathrm{C}$ burning, most of the star's energy is radiated via neutrinos. Since this mechanism of cooling is much more efficient, the fuel consumption increases rapidly.

After the last advanced burning stage, when $\mathrm{Si}$ is exhausted, the core consists mainly of iron peak nuclides. These nuclides exhibit the largest binding energy per nucleon and thus no more energy can be generated via fusion reactions. In other words, no other source of nuclear energy is available to the star. The structure of the massive star at this point is shown in the left half of Fig. 3. Meanwhile the mass of the core grows since the overlying burning shells produce more nuclear ashes. When the core grows to a mass near the Chandrasekhar limit $\left(M \approx 1.4 M_{\odot}\right)$, electron degeneracy pressure is unable to counteract gravity and the core starts to collapse. Complicated and poorly understood processes give rise to an outward moving shock, which initiates explosive burning while moving through the varius layers, and thus modifies the presupernova structure (see right half of Fig. 3). For more information, see, for example, Ref. [7]. In this brief review of nuclear astrophysics, we will mainly focus on the various hydrostatic burning stages, that gave rise to the onion shell structure of the massice star, as shown in the left half of Fig. 3.

\section{Nuclear Reactions}

The cross section of a nuclear reaction is defined as the number of interactions per time, divided by the number of incident particles per area and time, and divided by the number of target nuclei within the beam. The unit is barn, where 1 barn $\equiv 10^{-28} \mathrm{~m}^{2}$. For example, the estimated cross section for the weak reaction $p+p \rightarrow d+e^{+}+v$, which represents the first step in the pp chains (see below), is approximately $\sigma=8 \times 10^{-48} \mathrm{~cm}^{2}$ at a laboratory bombarding energy of 1 $\mathrm{MeV}$. If a measurement of this reaction would be performed using an intense $1 \mathrm{~mA}$ beam of protons, incident on a dense hydrogen target $\left(10^{20}\right.$ protons per $\left.\mathrm{cm}^{2}\right)$, then the expected reaction yield would amount only to 1 interaction in 6000 years! Clearly, such a measurement is beyond present experimental capabilities and hence this cross section needs to be estimated theoretically. In contrast, the next reaction in the pp chains, $d+p \rightarrow \rightarrow^{3} H e+\gamma$ has a cross section on the order of $3 \mu \mathrm{b}$ at a (proton) laboratory energy of $1 \mathrm{MeV}$ and is easily measured. However, the most important reactions to measure are usually the ones with the lowest cross sections since they regulate energy production and nucleosynthesis.

Cross section curves (cross section versus bombarding energy) come in many varieties. In the simplest case, the cross section of a charged-particle reaction drops dramatically with decreasing energy, but otherwise exhibits no structure. A good example is the cross section for ${ }^{16} \mathrm{O}(\mathrm{p}, \gamma){ }^{17} \mathrm{~F}$ below a center-of-mass energy of $2 \mathrm{MeV}$ (left side of Fig. 4). Sometimes the cross section exhibits 


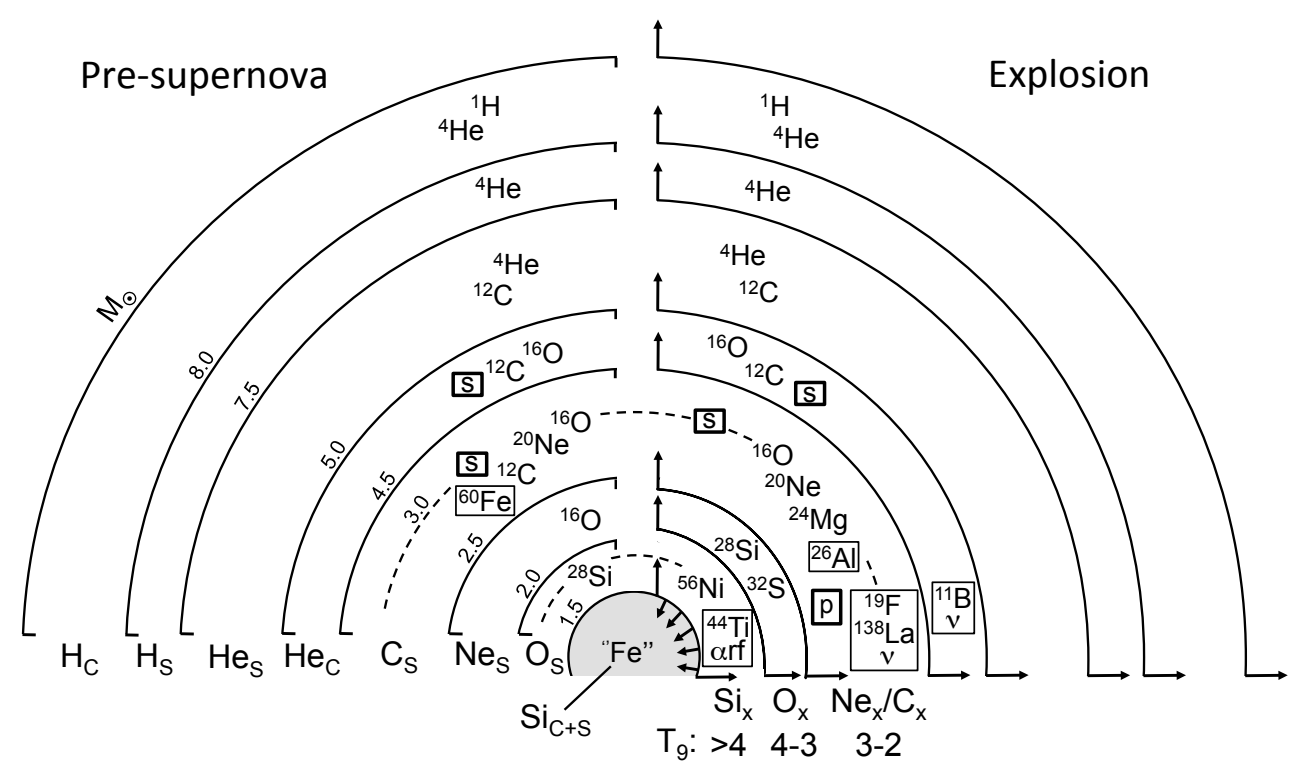

Figure 3: Structure of a $25 M_{\odot}$ star of solar metallicity, as predicted by one-dimensional, spherically symmetric models, shortly before and after core collapse (not to scale). Only the main constituents in each layer are shown. Minor constituents, among them important $\gamma$-ray emitters, are set in thin rectangles. (Left) Snapshot of pre-supernova structure. Nuclear burning takes place in thin regions (burning shells) at the interface of different compositional layers, where each burning shell migrated outward to the position indicated by the black lines. (Right) Explosive nucleosynthesis resulting from passage of the shock wave through overlying layers, giving rise to explosive burning of silicon $\left(\mathrm{Si}_{x}\right)$, oxygen $\left(\mathrm{O}_{x}\right)$ and neon-carbon $\left(\mathrm{Ne}_{x} / \mathrm{C}_{x}\right)$. This model is referred to as the onion shell structure of a massive star. For a detailed explanation of the figure, see Ref. [7].

a well-defined maximum. An example for such a behavior provides the ${ }^{13} \mathrm{C}(\mathrm{p}, \gamma){ }^{14} \mathrm{~N}$ reaction, which shows a maximum near $500 \mathrm{keV}$ in the center of mass (right side of Fig. 4). It is frequently stated that the transmission through the Coulomb barrier is responsible for the sharp drop in the cross section with decreasing energy, while the cross section maxima are identified as resonances. But how does the Coulomb barrier exactly explain the observation? And what is the origin of a resonance?

These questions can best be answered by considering a simple potential model for a nuclear reaction. We need essentially two pieces: an attractive (negative) square well potential of depth $V_{0}$ inside the nucleus $\left(r<R_{0}\right)$, and a repulsive (positive) square barrier potential of height $V_{1}$ for distances of $R_{0} \leq r<R_{1}$. The total energy of the incident particle, $E$, is less than the barrier height. An example for such a potential is shown in Fig. 5. The solution to the Schrödinger equation for each of the three regions I, II, III are well known and can be found in any introductory quantum mechanics textbook. In regions I and III, the solutions are in the form of complex exponentials, which represent a sine function. In region II, however, the solutions are given in terms of real exponentials. In the next step, the continuity condition is applied, that is, the wave function solutions and their derivatives must be continuous at the two boundaries $R_{0}$ and $R_{1}$. We obtain four equations and can solve for the intensity of the transmitted wave in region I, normalized to the intensity of the incident wave in region III. For relatively low energies one finds for this ratio, also called 

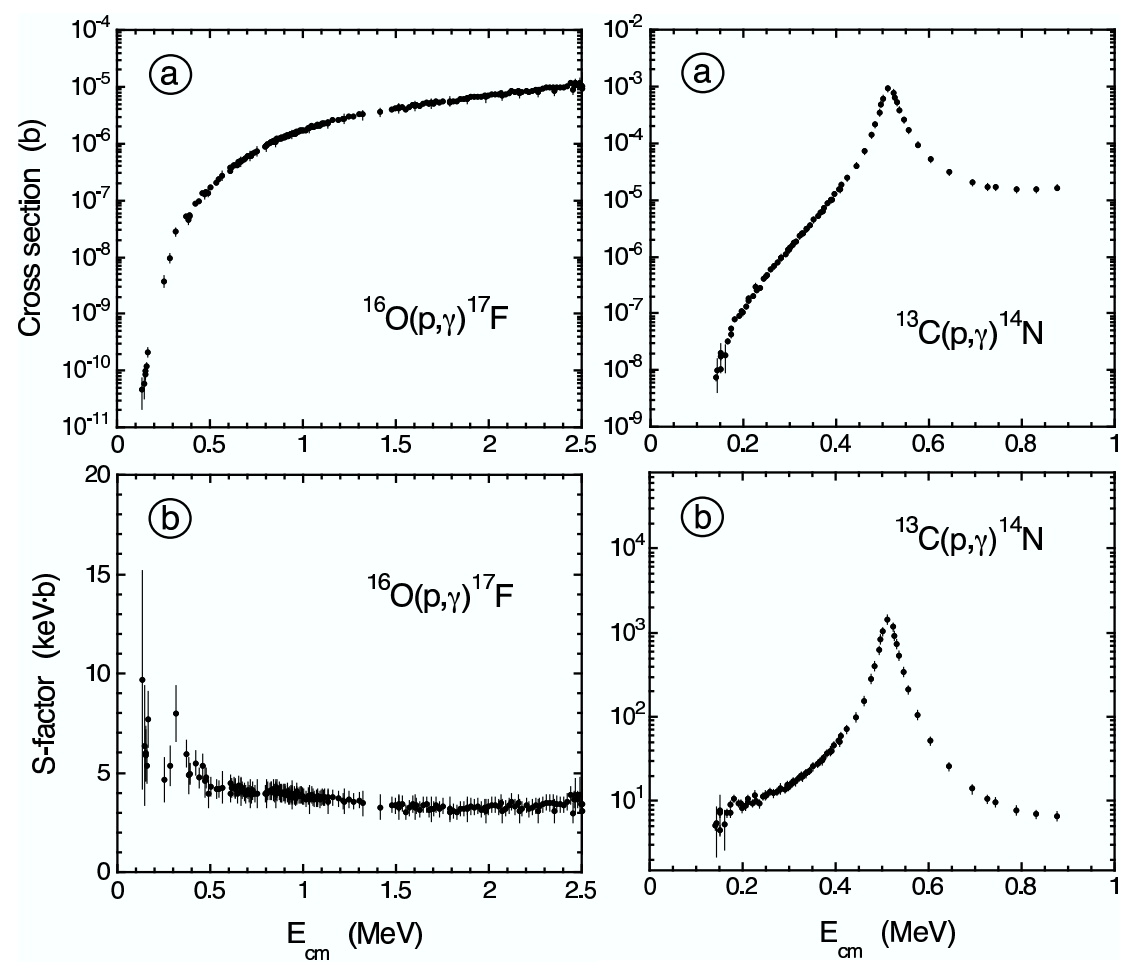

Figure 4: Textbook examples of nuclear reaction cross sections. (Left) ${ }^{16} \mathrm{O}(\mathrm{p}, \gamma){ }^{17} \mathrm{~F} ;($ Right $){ }^{13} \mathrm{C}(\mathrm{p}, \gamma){ }^{14} \mathrm{~N}$. Part (a) shows the cross section (in units of barn), while part (b) displays the astrophysical S-factor (in units of $\mathrm{keVb}$ ). Note the smooth energy dependence on the left, and the peak on the right. Figures adopted from Ref. [7].

transmission coefficient, after some algebra

$$
\hat{T} \approx e^{-(2 / \hbar) \sqrt{2 m\left(V_{1}-E\right)}\left(R_{1}-R_{0}\right)}
$$

where $m$ denotes the reduced mass. This result, referred to as the Tunnel effect, is remarkable and represents one of the most important quantum mechanical phenomena for charged particle reactions: although the incoming particle is classically not allowed to reach region I, there is a finite probability for tunneling through the barrier. Without this circumstance the world would be a very different place and life on Earth would certainly not exist. It is clear from Eq. (2.1) that the transmission coefficient depends very sensitively on the properties of the barrier. Its energy dependence, especially the sharp drop with decreasing energy, resembles that of the ${ }^{16} \mathrm{O}(\mathrm{p}, \gamma){ }^{17} \mathrm{~F}$ cross section.

We have not considered yet the full radial wave function solution of the Schrödinger equation for the three-dimensional case. In particular, we are interested in the ratio of the wave function intensities in regions I and III. It is now of advantage to express the wave function solutions in these regions as sine functions instead of complex exponentials. Otherwise we proceed as before: we apply the continuity condition and solve the system of four equations for this ratio. After some tedious algebra we find a rather lengthy analytical expression [7]. Interestingly, when plotting this function versus energy $E$ for certain values of the potential depth $V_{0}$, a well-defined maximum is 


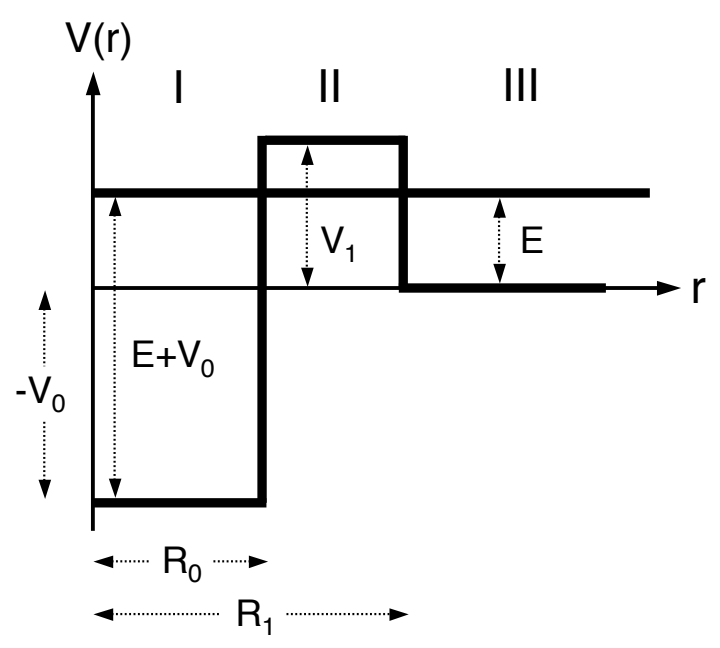

Figure 5: Three-dimensional square-well-plus-square-barrier potential, representing the simplest potential model for a charged-particle nuclear reaction. The potential properties are defined in the text. Note that for a real potential the transmission probability is only defined for the one-dimensional case. Thus for the calculation of the transmission coefficient we will assume that the figure represents a one-dimensional potential that extends to $-\infty$.

produced, while for other values of $V_{0}$ the resulting function closely reproduces the structureless energy-dependence of the transmission coefficient in Eq. (2.1). Note that by changing the potential depth $V_{0}$ we are changing the wavelength in the nuclear interior (region I) and, for discrete values of $V_{0}$, the wave function amplitude in the nuclear interior becomes relatively large. This describes, in the simplest case, the origin of a well-defined cross section maximum: a resonance results from favorable wave function matching conditions at the nuclear boundary.

In a more realistic situation we need to replace the simple square barrier by the Coulomb potential (if we disregard the centripetal barrier for the moment). The Coulomb potential has a more complicated shape, but we may approximate it by dividing this potential into many thin square barriers. The transmission coefficient for the Coulomb potential is then given by the product of the transmission coefficients for all of the square barriers. If we let the number of square barriers become very large $(n \rightarrow \infty)$, the transmission coefficient for the Coulomb potential can be found analytically. For very low energies we find

$$
\hat{T} \approx \exp \left(-\frac{2 \pi}{\hbar} \sqrt{\frac{m}{2 E}} Z_{0} Z_{1} e^{2}\right) \equiv e^{-2 \pi \eta}
$$

where $Z_{0}$ and $Z_{1}$ are the charges of the interacting nuclei and $e$ is the elementary charge. This function reveals a $1 / \sqrt{E}$ dependence in the exponent and is referred to as the Gamow factor. It is frequently used in nuclear astrophysics to define a useful quantity, called the astrophysical $S$ factor, via the relation $S(E) \equiv E \sigma(E) \exp (2 \pi \eta)$ : division by the Gamow factor removes from the cross section $\sigma(E)$ the strong Coulomb barrier transmission probability and produces a function, $S(E)$, that is more manageable, for example, in theoretical extrapolations to very low energies. The astrophysical S-factors for the ${ }^{16} \mathrm{O}(\mathrm{p}, \gamma){ }^{17} \mathrm{~F}$ and ${ }^{13} \mathrm{C}(\mathrm{p}, \gamma){ }^{14} \mathrm{~N}$ reactions, which are shown in the bottom half of Fig. 4, emphasize this point. 
In formal reaction theory, a simple equation describing a single isolated resonance can be derived. It is referred to as Breit-Wigner formula and is given by

$$
\sigma_{\mathrm{BW}}(E)=\frac{\lambda^{2} \omega}{4 \pi} \frac{\Gamma_{a} \Gamma_{b}}{\left(E_{r}-E\right)^{2}+\Gamma^{2} / 4}
$$

where $\lambda$ is the de Broglie wavelength, $\omega$ is a factor containing angular momenta, $E_{r}$ is the resonance energy, $\Gamma_{i}$ are the resonance partial widths of entrance and exit channel, and $\Gamma$ is the total resonance width given by the sum of all partial widths. The above equation is the single most important expression describing a resonance and it is frequently used in nuclear astrophysics in many applications: (i) for fitting cross section data to extract resonance parameters; (ii) for deriving the narrow-resonance reaction rate (see below); (iii) for extrapolating cross sections to energy regions were no measurements exist; and (iv) for calculating the experimental resonance yield when the resonance cannot be resolved experimentally.

A partial width describes the probability (in energy units) per unit time for formation or decay of a resonance. For example, the partial width for forming a resonance via proton absorption, or for decay of a resonance via proton emission, is given by the expression

$$
\Gamma_{\lambda c}=2 \frac{\hbar^{2}}{m R^{2}} P_{c} C^{2} S \theta_{p c}^{2}
$$

Apart from a constant factor involving the reduced mass $m$ and the nuclear radius $R$, the proton partial width is given by the product of three distinct probabilities: first, the probability that the nucleons will arrange themselves in a target-plus-single-particle configuration (spectroscopic factor, $C^{2} S$ ); second, the probability that a proton will appear at the nuclear boundary (dimensionless reduced single-particle width, $\theta_{p c}^{2}$ ); and, finally, the probability that the proton will penetrate the Coulomb and centripetal barriers (penetration factor, $P_{c}$ ). The third factor, which can be computed precisely from Coulomb wave functions, is strongly energy-dependent. The second factor can also be computed numerically [9]. The great untility of Eqs. (2.3)-(2.4) becomes now apparent: if the spectroscopic factor, $C^{2} S$, which is a nuclear structure quantity, can be estimated by different means, for example, using transfer reaction studies, then the partial width can be calculated and the cross section be estimated in a straightforward manner, despite the fact that the reaction cross section has not been measured directly. Clearly, in many cases the cross section cannot be measured directly, either because the Coulomb barrier transmission probability is too small or perhaps because the target is short-lived. Consequently, such indirect methods of estimating the cross section become a crucial tool in nuclear astrophysics.

\section{Thermonuclear Reactions}

In a stellar plasma, the kinetic energy for a nuclear reaction derives from the thermal motion of the participating nuclei. Hence, the interaction is referred to as thermonuclear reaction. The thermonuclear reaction rate (the number of reactions per unit time and unit volume) for a reaction $0+1 \rightarrow 2+3$ is given by $r_{01}=N_{0} N_{1}\langle\sigma \mathrm{v}\rangle_{01}$, where $N_{i}$ are the number densities of the interacting nuclei and $\langle\sigma \mathrm{v}\rangle_{01}$ is the reaction rate per particle pair, which is equal to the integral over the product of velocity, cross section, and velocity probability density. In most cases of practical interest, the 
latter function is given by the Maxwell-Boltzmann distribution. Thus the reaction rate per particle pair can be written as

$$
\langle\sigma \mathrm{v}\rangle_{01}=\left(\frac{8}{\pi m_{01}}\right)^{1 / 2} \frac{1}{(k T)^{3 / 2}} \int_{0}^{\infty} E \sigma(E) e^{-E / k T} d E
$$

where $m_{01}$ is the reduced mass, $k$ the Boltzmann constant and $T$ the plasma temperature. For a given temperature the reaction rate is precisely determined if the nuclear reaction cross section, $\sigma(E)$, is known.

At this point it is worthwhile to note that a given nuclear reaction occurring in the stellar plasma can rarely be considered as an isolated interaction. Consider, for example, the species ${ }^{25} \mathrm{Al}$ at an elevated temperature. It may be destroyed in several different ways: via $\beta^{+}$-decay to ${ }^{25} \mathrm{Mg}$, via proton capture to ${ }^{26} \mathrm{Si}$, via photodisintegration to ${ }^{24} \mathrm{Mg}$, and so on. On the other hand, ${ }^{25} \mathrm{Al}$ is produced via the $\beta^{+}$-decay of ${ }^{25} \mathrm{Si}$, via proton capture on ${ }^{24} \mathrm{Mg}$, via photodisintegration of ${ }^{26} \mathrm{Si}$, and so on. The abundance evolution of ${ }^{25} \mathrm{Al}$ during the nucleosynthesis is then given by a differential equation that accounts for all destruction and production mechanisms. Such a differential equation needs to be written for all species participating in the nuclear burning. Thus one ends up with a system of coupled differential equations, called a nuclear reaction network. A good introduction of how to solve this system numerically can be found in Dave Arnett's book [10].

It is interesting to investigate Eq. (3.1) in more detail by considering a few extreme examples. We start with the simplest case, i.e., a nearly constant S-factor, $S_{0}$, as shown in Fig. 4 for the ${ }^{16} \mathrm{O}(\mathrm{p}, \gamma){ }^{17} \mathrm{~F}$ reaction. This situation is usually referred to as "non-resonant", which however leads to considerable misunderstandings since the formalism also applies to slowly varying resonance tails, as will be seen below. Substitution of the S-factor definition (see above) into Eq. (3.1) shows immediately that the reaction rate depends, apart from the magnitude of $S_{0}$, on the integral over the product of Gamow and Boltzmann factors, $e^{-2 \pi \eta} e^{-E / k T}$. The situation is shown in Fig. 6 for the ${ }^{12} \mathrm{C}(\alpha, \gamma){ }^{16} \mathrm{O}$ reaction at a stellar temperature of $0.2 \mathrm{GK}$. It is apparent that the star does not burn at high energies where the cross section (dash-dotted line) is large (since the number of particles with such energies is vanishingly small); neither does the star burn at very small energies where the number of particles (dashed line) is at maximum (since the cross section is vanishingly small). Rather, in a plasma most nuclear reactions occur at energies where the product $e^{-2 \pi \eta} e^{-E / k T}$ is at maximum. This well-defined energy window is referred to as the Gamow peak (solid lines) and represents the effective energy window of stellar burning for a given nuclear reaction.

When the Gamow peak is plotted for a given temperature, but for different target-projectile combinations (implying different projectile and target charges and hence different Coulomb barrier heights), a few important observations can be made. For increasing charges $Z_{0}$ and $Z_{1}$ : (i) the Gamow peak shifts to higher energies; (ii) the Gamow peak becomes broader; and most importantly, (iii) the area under the Gamow peak decreases dramatically. In other words, for a mixture of different nuclides in a stellar plasma at given temperature, those reactions with the smallest Coulomb barrier generate most of the nuclear energy and are consumed most rapidly. This aspect is of paramount importance for the star since it explains the occurrence of well-defined stellar burning stages (see Sec. 4).

Next, we will consider a narrow resonance. Several different definitions for a narrow resonance can be found in the literature, but all lead to problems if used uncritically. For the sake 

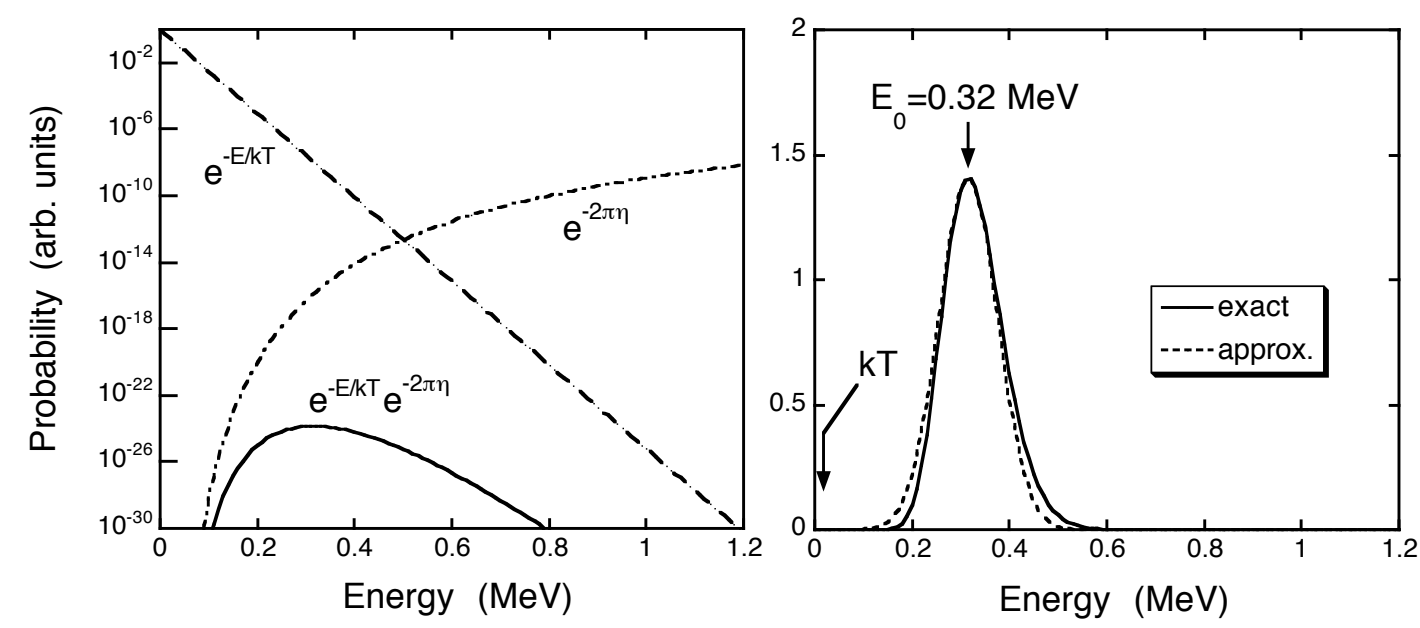

Figure 6: (Left) Maxwell-Boltzmann factor $\left(e^{-E / k T}\right.$; dashed line) and Gamow factor $\left(e^{-2 \pi \eta} ;\right.$ dashed-dotted line) versus energy for the ${ }^{12} \mathrm{C}(\alpha, \gamma){ }^{16} \mathrm{O}$ reaction at a temperature of $T=0.2 \mathrm{GK}$. The product $e^{-E / k T} e^{-2 \pi \eta}$, referred to as the Gamow peak, is shown as solid line. (Right) The same Gamow peak shown on a linear scale (solid line). The maximum occurs at $E_{0}=0.32 \mathrm{MeV}$, while the maximum of the Maxwell-Boltzmann distribution at this temperature is located at $k T=0.017 \mathrm{MeV}$. The dotted line shows the Gaussian approximation of the Gamow peak. Adopted from Ref. [7].

of simplicity, let us assume that a narrow resonance implies constant partial widths over the total width of the resonance. Substitution of Eq. (2.3) into Eq. (3.1) yields immediately $\langle\sigma \mathrm{v}\rangle=$ $[(2 \pi) /(m k T)]^{3 / 2} \hbar^{2} e^{-E_{r} / k T} \omega \gamma$. The product $\omega \gamma \equiv \omega \Gamma_{a} \Gamma_{b} / \Gamma$ is proportional to the area under the narrow-resonance cross section curve and thus is called resonance strength (with units of energy). Note that the resonance energy enters exponentially in the above reaction rate expression and thus needs to be determined rather precisely. Otherwise the resulting uncertainty of the reaction rate becomes large.

In many cases the energy-dependence of the partial widths over the total width of the resonance cannot be disregarded. Such broad-resonance reaction rates need to be treated with care. Although approximate expressions exist in the literature, it is safer to substitute Eq. (2.3) into Eq. (3.1) and evaluate the integral numerically. Depending on the location of the broad resonance with respect to the Gamow peak, there are in general two contributions to the total reaction rate. First, the contribution corresponding to the narrow resonance reaction rate, which arises only from the region near the resonance energy (as is apparent from the factor $e^{-E_{r} / k T}$ ). Second, the contribution from the smoothly varying tail of the resonance. If the broad resonance is located outside the Gamow peak, then in most cases the resonance tail makes a far larger contribution than what is calculated from the narrow resonance expression. Plotting such reaction rates versus temperature frequently reveals a kink because the narrow resonance and broad resonance reaction rates have different temperature dependences.

To evaluate the total rate of a single reaction, many different contributions need to be taken into account: narrow and broad resonances, non-resonant processes, subthreshold resonances, cross section continua, interferences between different amplitudes, etc. Every single reaction represents 
a special case and the evaluation process is usually tedious. Evaluations of experimental reaction rates have been provided by Willy Fowler and collaborators for many years, with their last evaluation (covering the $A=1-30$ target mass range) published in 1988 [11]. A European effort, by the NACRE collaboration, resulted in an updated reaction rate evaluation in 1999 [12], while another evaluation including for the first time radioactive target nuclei was published in 2001 [13]. A modern evaluation that utilizes Monte Carlo techniques for estimating recommended experimental rates and associated uncertainties can be found in Refs. [14, 15, 16, 17].

\section{Stellar Burning Stages}

\subsection{Hydrostatic hydrogen burning}

Hydrostatic burning of $\mathrm{H}$ occurs near $T=15.6 \mathrm{MK}$ in the center of the Sun, in the range of $T=8-55 \mathrm{MK}$ in the cores of other stars, depending on their mass, and at $T=45-100 \mathrm{MK}$ in the $\mathrm{H}$ burning shell of AGB stars. If only $\mathrm{H}$ and $\mathrm{He}$ are available as fuel, without the presence of heavier nuclides, then the stellar core generates nuclear energy via the pp chains. These are shown schematically in Fig. 7a. All chains fuse effectively four protons to one ${ }^{4} \mathrm{He}$ nucleus and thereby generate an energy of $26.7 \mathrm{MeV}$. Furthermore, at low temperatures, all chains involve nonresonant reactions only. Each chain starts with the $\mathrm{p}\left(\mathrm{p}, \mathrm{e}^{+} v\right) \mathrm{d}$ reaction, which cannot be measured yet directly at the relevant energies (see Sec. 1). The absolute magnitude of this cross section is influenced by the weak interaction. Fortunately, the different factors that determine the S-factor can be estimated theoretically with substantial confidence [18]. The present reaction rate uncertainty amounts to a few percent only [12], which is significantly smaller compared to rate uncertainties of most directly measured stellar fusion reactions. Several other reactions that are part of the pp chains have been measured directly, most recently the $\mathrm{d}(\mathrm{p}, \gamma){ }^{3} \mathrm{He},{ }^{3} \mathrm{He}\left({ }^{3} \mathrm{He}, 2 \mathrm{p}\right) \alpha$ and ${ }^{3} \mathrm{He}(\alpha, \gamma){ }^{7} \mathrm{Be}$ reactions by the LUNA collaboration [19].

In many situations small amounts of ${ }^{12} \mathrm{C}$ and ${ }^{16} \mathrm{O}$ will be present in the stellar plasma and these nuclei will participate in the stellar burning. For example, ${ }^{12} \mathrm{C}$ captures a proton yielding ${ }^{13} \mathrm{~N}$, which in turn, $\beta$-decays to ${ }^{13} \mathrm{C}$. This nuclide captures another proton, and then another one to yield ${ }^{15} \mathrm{O}$, which $\beta$-decays to ${ }^{15} \mathrm{~N}$. At this point something important occurs: rather than capturing another proton, ${ }^{15} \mathrm{~N}$ prefers to undergo a $(\mathrm{p}, \alpha)$ reaction, producing again ${ }^{12} \mathrm{C}$. The interesting point here is that by completing one cycle, four protons have been fused to one ${ }^{4} \mathrm{He}$ nucleus, while the heavy seed nucleus has been recovered. Thus, ${ }^{12} \mathrm{C}$ acts as a catalyst, implying that even small amounts of CNO material can give rise to a large nuclear energy generation. A small leakage of material via ${ }^{15} \mathrm{~N}(\mathrm{p}, \gamma){ }^{16} \mathrm{O}$ initiates different cyclic reaction sequences. They are collectively referred to as $C N O$ cycles and are shown in Fig. 7b. In each case, the $(\mathrm{p}, \alpha)$ reaction is favored over the $(\mathrm{p}, \gamma)$ reaction at the branching points ${ }^{15} \mathrm{~N},{ }^{17} \mathrm{O},{ }^{18} \mathrm{O}$ and ${ }^{19} \mathrm{~F}$, which is a necessary condition for a reaction cycle to occur. The first cycle, called CNOI cycle, is the most important one. It is governed by ${ }^{14} \mathrm{~N}(\mathrm{p}, \gamma){ }^{15} \mathrm{O}$, since it is by far the slowest interaction among the reactions and $\beta$-decays. This reaction has been measured both at the LUNA facility (deep underground in Gran Sasso) [19], and at the LENA facility (at sea-level in our laboratory) [20]. It was found that the new reaction rate deviates from the previous one [12] by about a factor of $\approx 2$. As a consequence, the ages of globular clusters (see Sec. 1), obtained by fitting the turn-off point in the Hertzsprung-Russell diagram to stellar models 


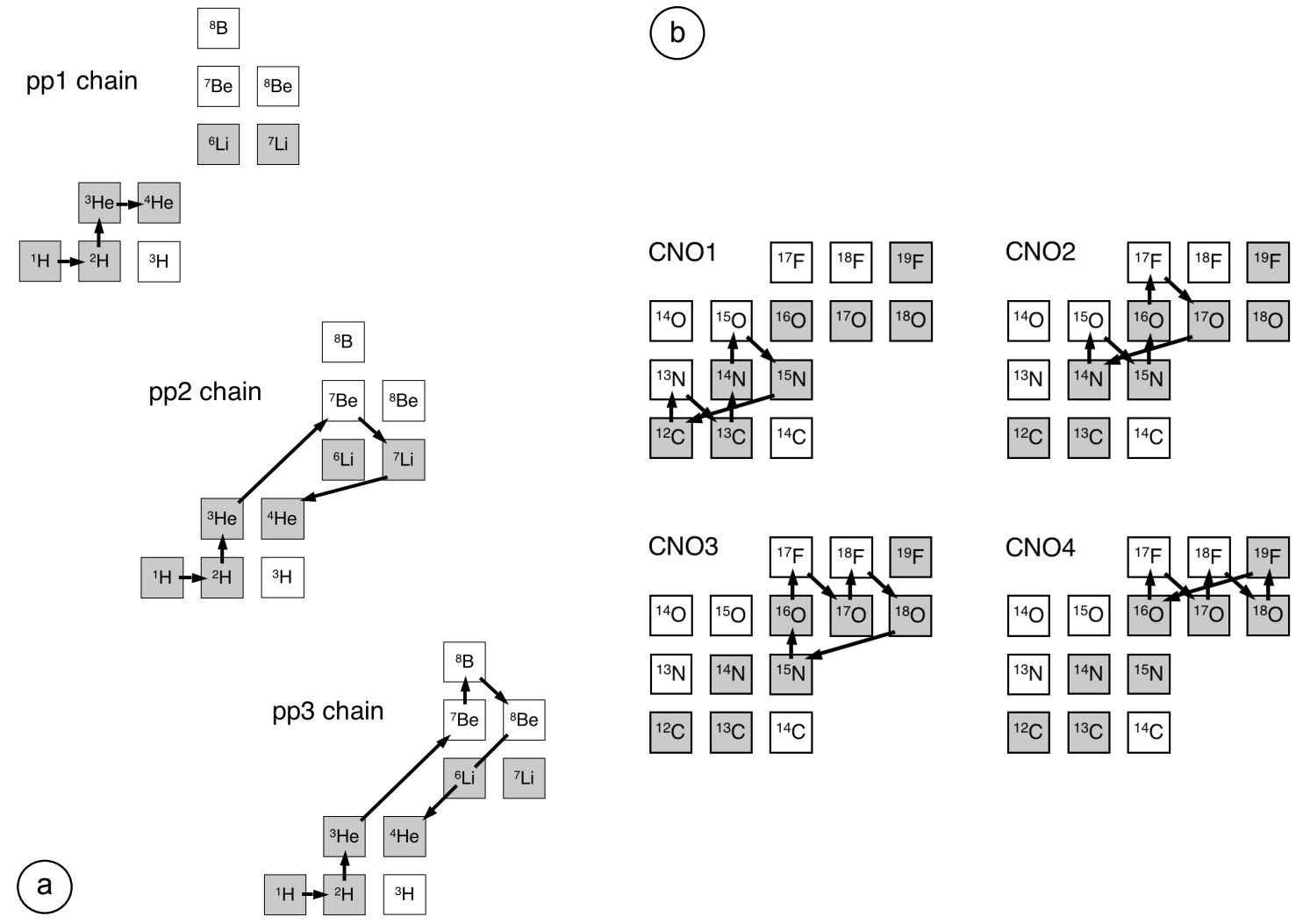

Figure 7: The pp chains (part a) and the CNO cycles (part b) shown schematically in the chart of the nuclides. Each arrow represents a specific interaction, connecting an initial with a final nuclide. Stable nuclides are shown as shaded squares. The proton and neutron numbers increase in the vertical and horizontal directions, respectively. Adopted from Ref. [7].

of low-mass stars, changed by about $1 \mathrm{~Gy}$ ! This again emphasizes the dramatic impact of accurate cross section measurements on stellar models and on cosmological questions.

The solar carbon isotopic number abundance ratio is ${ }^{13} \mathrm{C} /{ }^{12} \mathrm{C}=0.01$, while the $\mathrm{CNO} 1$ cycle equilibrium ratio amounts to ${ }^{13} \mathrm{C} /{ }^{12} \mathrm{C}=0.25$. Many stars that burn $\mathrm{H}$ via the $\mathrm{CNO} 1$ cycle have observed ratios between these two values, while a few stars even come close to the equilibrium value. This implies that a significant fraction of these stars' hydrogen envelope has been cycled through regions that experienced equilibrium operation of the $\mathrm{CNO} 1$ cycle. From the latest reaction cross sections, assuming a solar composition, one finds that near $T=20 \mathrm{MK}$ the CNO1 cycle takes over from the pp1 chain as the main energy-generating process. Thus it is found that about $90 \%$ of the Sun's energy generation originates from the pp1 chain. The CNO cycles occurring in AGB stars are predicted to be a major source of ${ }^{13} \mathrm{C}$ and ${ }^{14} \mathrm{~N}$ in the Universe.

\subsection{Hydrostatic helium burning}

Hydrostatic burning of helium, for example, in massive stars takes place in the temperature range $T=0.1-0.4 \mathrm{GK}$. The important reactions are shown in Fig. 8. Helium burning starts with the fusion of two $\alpha$-particles. However, the composite nucleus ${ }^{8} \mathrm{Be}$ lives for only $\approx 10^{-16} \mathrm{~s}$ and 


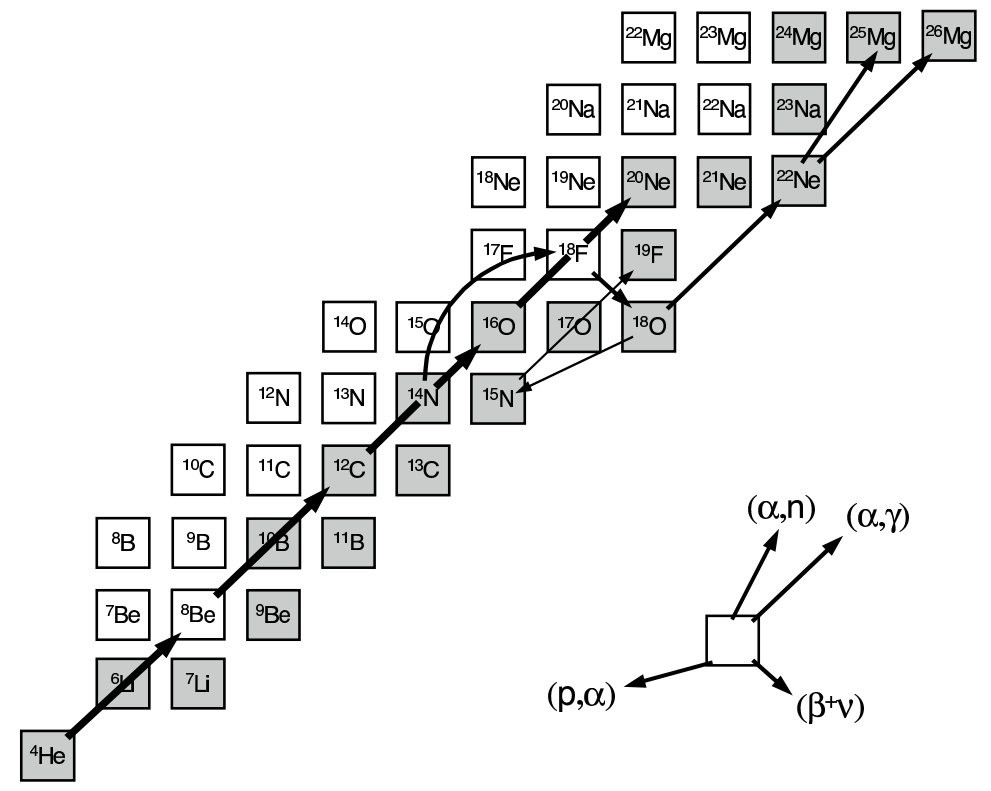

Figure 8: Representation of helium-burning reactions in the chart of the nuclides. Stable nuclides are shown as shaded squares. The key relates an arrow to a specific interaction. The $3 \alpha$ reaction and the $(\alpha, \gamma)$ reactions on ${ }^{12} \mathrm{C}$ and ${ }^{16} \mathrm{O}$ are displayed as thick arrows. Other helium-burning reactions are shown as thinner arrows. The reaction ${ }^{14} \mathrm{~N}(\alpha, \gamma){ }^{18} \mathrm{~F}$ is represented by an arc for reasons of clarity. Adopted from Ref. [7].

decays back into two $\alpha$-particles. Nevertheless, after a given time a tiny equilibrium abundance of ${ }^{8} \mathrm{Be}$ builds up, sufficient to allow for capture of a third $\alpha$-particle to form stable ${ }^{12} \mathrm{C}$. This process is referred to as triple- $\alpha$ reaction. It was pointed out by Fred Hoyle [21] that this process would be too slow to account for the fusion of ${ }^{12} \mathrm{C}$, unless a resonance exists right above the ${ }^{8} \mathrm{Be}+\alpha$ threshold, which furthermore must be formed without inhibition by the centripetal barrier (i.e., it has to be a s-wave resonance). A few years later this level in ${ }^{12} \mathrm{C}$, referred to since as the Hoyle state, was experimentally verified. The prediction and the subsequent verification of this state reflects the marvelous interplay of astrophysics and nuclear physics. The triple- $\alpha$ reaction is a sequential three-body interaction and thus has not been measured in the laboratory. From experimental knowledge of the nuclear masses and partial widths involved, the reaction rate can be estimated fairly accurately. Present uncertainties amount to about $\pm 15 \%$ [12], a remarkably small value for a process that has not be measured directly.

Helium burning continues via the ${ }^{12} \mathrm{C}(\alpha, \gamma){ }^{16} \mathrm{O}$ reaction. There is no resonance near and above the $\alpha$-particle threshold in ${ }^{16} \mathrm{O}$ and thus this process must proceed via broad-resonance tails (including subthreshold resonances) and direct mechanisms. These amplitudes may interfere, causing problems in the extrapolation of the S-factor to the astrophysically important energy range, which at present is not accessible experimentally. The rate of this reaction is of great importance, since it determines the ${ }^{12} \mathrm{C}$ to ${ }^{16} \mathrm{O}$ abundance ratio at the end of helium burning. This abundance ratio sensitively influences not only all the subsequent hydrostatic burning stages in massive stars, but also the explosive burning, and the nature of the remnant (neutron star or black hole) left behind after the core collapse. At present the reaction rate is uncertain by $\pm 35 \%$, to be conservative, and a more precise rate is highly desirable. The subsequent ${ }^{16} \mathrm{O}(\alpha, \gamma){ }^{20} \mathrm{Ne}$ reaction is very slow, which 
explains the survival of ${ }^{16} \mathrm{O}$ during helium burning. The main products at the end of helium burning are ${ }^{12} \mathrm{C}$ to ${ }^{16} \mathrm{O}$.

If ${ }^{14} \mathrm{~N}$ is present in the stellar plasma (from the $\mathrm{CNO}$ cycle operation during the preceding $\mathrm{H}$ burning stage; Sec. 4.1), then the reaction sequence ${ }^{14} \mathrm{~N}(\alpha, \gamma){ }^{18} \mathrm{~F}\left(\beta^{+} v\right)^{18} \mathrm{O}(\alpha, \gamma){ }^{22} \mathrm{Ne}$ can be initiated. The subsequent ${ }^{22} \mathrm{Ne}(\alpha, \mathrm{n})^{25} \mathrm{Mg}$ reaction is the main neutron source towards the end of helium burning, and gives rise to the weak component of the s-process. Hydrostatic helium burning is predicted to be a major source of ${ }^{12} \mathrm{C},{ }^{16} \mathrm{O},{ }^{18} \mathrm{O}$ and ${ }^{22} \mathrm{Ne}$ in the Universe.

\subsection{Hydrostatic carbon, neon, oxygen, and silicon burning}

As we have seen, after the end of He burning the core consists mainly of ${ }^{12} \mathrm{C}$ and ${ }^{16} \mathrm{O}$. Since the combination of two ${ }^{12} \mathrm{C}$ nuclei has the lowest Coulomb barrier, the next most likely nuclear fuel to ignite is ${ }^{12} \mathrm{C}$. For the first time in the life of the star, a heavy-ion fusion reaction, ${ }^{12} \mathrm{C}+{ }^{12} \mathrm{C}$, is defining a burning stage, which is referred to as carbon burning. Typical core temperatures amount to $T=0.6-1.0 \mathrm{GK}$. There are three possible primary reactions, ${ }^{12} \mathrm{C}\left({ }^{12} \mathrm{C}, \mathrm{p}\right){ }^{23} \mathrm{Na},{ }^{12} \mathrm{C}\left({ }^{12} \mathrm{C}, \alpha\right){ }^{20} \mathrm{Ne}$ and ${ }^{12} \mathrm{C}\left({ }^{12} \mathrm{C}, \mathrm{n}\right){ }^{23} \mathrm{Mg}$. The released light particles undergo several secondary reactions involving newly formed nuclei, among them ${ }^{25} \mathrm{Mg}(\mathrm{p}, \gamma)^{26} \mathrm{Al}$. The main ashes of $\mathrm{C}$ burning are ${ }^{16} \mathrm{O}$, which has not participated much in the nuclear activity, and ${ }^{20} \mathrm{Ne}$. The primary reaction, ${ }^{12} \mathrm{C}+{ }^{12} \mathrm{C}$, populates levels in the compound nucleus ${ }^{24} \mathrm{Mg}$ near $14 \mathrm{MeV}$ excitation energy. This energy region exhibits a very high level density, with many broad and overlapping states. Therefore, we expect the S-factor to be a smooth function of energy. However, experiments have uncovered many sharp maxima in the S-factor curve, even near astrophysically important energies [19]. A satisfactory reaction model to explain this structure is lacking at present. This is problematic since the available data do not cover the entire astrophysically important energy region and hence we have to rely on rather uncertain extrapolations.

After $\mathrm{C}$ burning, when the temperature in the core reaches values of $T=1.2-1.8 \mathrm{GK}$, the most likely process to occur is the photodisintegration of ${ }^{20} \mathrm{Ne}$ via the (primary) ${ }^{20} \mathrm{Ne}(\gamma, \alpha){ }^{16} \mathrm{O}$ reaction. For the first time in the life of the star, a photodisintegration defines a burning stage. The released $\alpha$-particles (including proton and neutrons at a slightly later time) initiate a number of secondary reactions and the evolving reaction network is referred to as neon burning. Although the primary reaction is endothermic (it consumes energy), together with the secondary reactions there is a net production of nuclear energy for each ${ }^{20} \mathrm{Ne}$ nucleus destroyed. The main nuclear ash of $\mathrm{Ne}$ burning is ${ }^{16} \mathrm{O}$.

The core contracts further after Ne burning until temperatures of $T=1.5-2.7 \mathrm{GK}$ are produced. At this stage another (primary) heavy ion reaction, ${ }^{16} \mathrm{O}+{ }^{16} \mathrm{O}$, initiates a burning stage, called oxygen burning. The temperatures are so high that many exit channels are open, some of which even involve the emission of three particles. The light particles then initiate a number of secondary reactions. It is interesting to note that unlike the case of ${ }^{12} \mathrm{C}+{ }^{12} \mathrm{C}$, the ${ }^{16} \mathrm{O}+{ }^{16} \mathrm{O}$ reaction exhibits a smooth energy-dependence of the S-factor, as expected. Nevertheless, the S-factor data at the lowest measured energies are in poor agreement and, furthermore, the branching ratios for the different exit channels need also be known to better accuracy. Clearly, more laboratory work is required. The main ashes of $\mathrm{O}$ burning are ${ }^{28} \mathrm{Si}$ and, to a somewhat lesser extent, ${ }^{32} \mathrm{~S}$.

After O burning, the core contracts until temperatures of $T=2.8-4.1 \mathrm{GK}$ are reached. At this point the photodisintegration ${ }^{28} \mathrm{Si}(\gamma, \alpha){ }^{24} \mathrm{Mg}$ initiates another burning stage, called silicon burning. 
As was the case before, the released light particles give rise to a network of secondary reactions, but on a much grander scale than during Ne burning. In essence, during this photodisintegration rearrangement, less tightly bound nuclides are photodisintegrated and the released protons, neutrons, and $\alpha$-particles are captured to synthesize more tightly bound species. Many reactions achieve equilibrium during $\mathrm{Si}$ burning. It is important to note that, once equilibrium has been achieved between two species, $A$ and $B$, the thermonuclear rates of the reactions $A \rightarrow B$ and $B \rightarrow A$ are entirely irrelevant for the nuclear transformations. This can be shown by applying the Saha statistical equation and the reciprocity theorem of nuclear reactions [7]. All that is needed (apart from some less important factors) is the $Q$-value of the $A \rightarrow B$ reaction.

Numerical network calculations show that two major groups of nuclides form equilibrium clusters (also called quasiequilibrium clusters): one forms around ${ }^{28} \mathrm{Si}$ (and extends up to $A \approx 40$ ), the other one forms around the iron peak nuclei (starting near $A \approx 50$ ). These two clusters are only weakly linked by other reactions and thus are not in mutual equilibrium for a significant amount of time during Si burning. One major reason is that ${ }^{40} \mathrm{Ca}$ is a doubly-magic nucleus (with 20 protons and 20 neutrons), so that the capture of a light particle is energetically unfavorable. Hence, the product nucleus is quickly photodisintegrated back to ${ }^{40} \mathrm{Ca}$. Nevertheless, given enough time a physical system will seek a state of most favorable energy and, via reactions in the $A \approx 40-50$ range, the abundances of the $\mathrm{Si}$ cluster species decline with time in favor of those in the iron peak cluster. The fact that a plasma composed of iron peak nuclei is energetically much more favorable than one of silicon is demonstrated in Fig. 9, showing the binding energy per nucleon versus the mass number of the nuclide. Binding energies per nucleon between 8.6 and $8.8 \mathrm{MeV}$ are achieved by iron peak species, while the value for ${ }^{28} \mathrm{Si}$ amounts to $8.45 \mathrm{MeV}$. Detailed reaction network calculations show that at the end of Si burning the most abundant product is ${ }^{56} \mathrm{Fe}$.

\subsection{Nuclear statistical equilibrium}

As ${ }^{28} \mathrm{Si}$ disappears in the core at the end of Si burning, the temperature increases until all non-equilibrated reactions come into equilibrium. Now one large cluster stretches from protons, neutrons, and $\alpha$-particles all the way to the iron peak, and the reaction network attains nuclear statistical equilibrium (NSE). The abundance of any nuclide in nuclear statistical equilibrium can be calculated from a repeated application of the Saha equation. For species ${ }_{\pi}^{A} Y_{V}$, with mass number $A, \pi$ protons and $v$ neutrons, one finds for the number abundance

$$
N_{Y}=N_{p}^{\pi} N_{n}^{v} \frac{1}{\theta^{A-1}}\left(\frac{M_{Y}}{M_{p}^{\pi} M_{n}^{v}}\right)^{3 / 2} \frac{g_{Y}}{2^{A}} G_{Y}^{\text {norm }} e^{B(Y) / k T}
$$

where $\theta$ is a constant [7], $N_{p}$ and $N_{n}$ are the number abundances of free protons and neutrons, respectively, $M_{i}$ is the nuclear mass of species $i, g_{Y}$ is the statistical weight (which depends on the spin of $Y$ ), $G_{Y}^{\text {norm }}$ is the normalized partition function (which depends on energies and spins of excited levels in $Y$ ), and $B(Y)$ is the binding energy. Note that in the above equation reaction rates are absent, which of course is expected since the reaction network has achieved equilibrium.

Provided that the nuclear physics information on binding energies, spins, and excitation energies is available, the abundance of any nuclide in nuclear statistical equilibrium is determined by only three independent parameters: temperature, density, and neutron excess. The latter parameter 


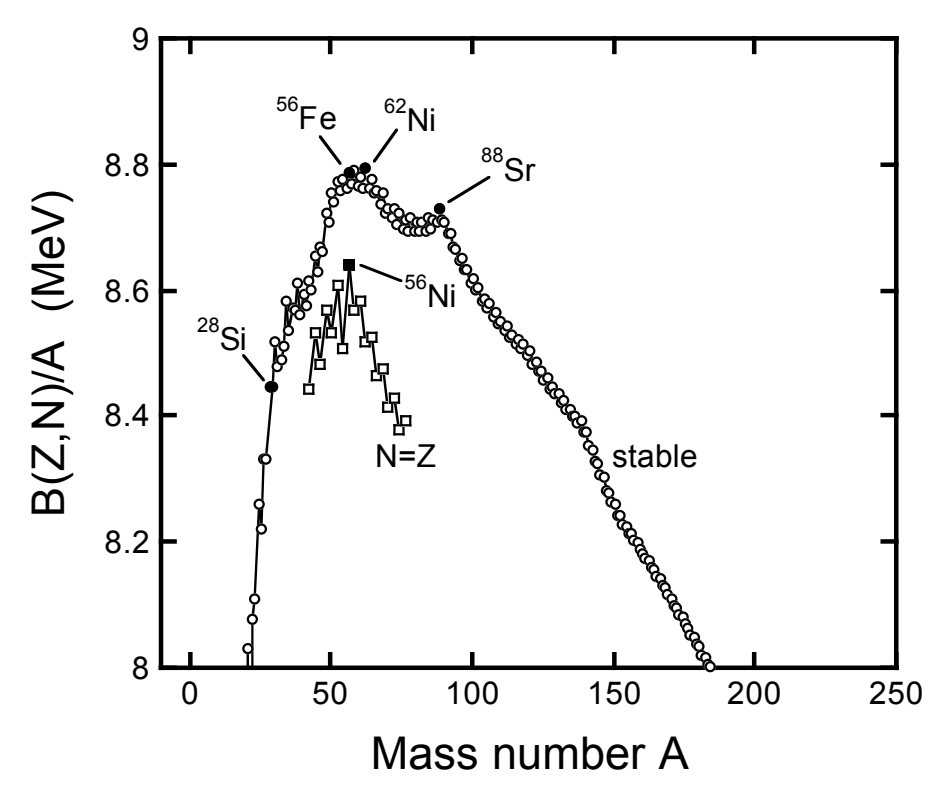

Figure 9: Experimental binding energy per nucleon, $B(Z, N) / A$, versus mass number, $A$. The round symbols correspond to the most tightly bound nuclide of a given mass number; the nuclide with the largest binding energy per nucleon is ${ }^{62} \mathrm{Ni}(B / A=8.795 \mathrm{MeV})$; the square symbols show $B(Z, N) / A$ values for $N=Z$ nuclides above $A=40$, which are all radioactive; the $N=Z$ species with the largest binding energy per nucleon is ${ }^{56} \mathrm{Ni}(B / A=8.643 \mathrm{MeV})$. Adopted from Ref. [7].

is defined as $\eta \equiv \sum_{i}\left(v_{i}-\pi_{i}\right) X_{i} / M_{i}$, with $X_{i}$ the mass fraction. The sum runs over all species $i$ present in the plasma. The neutron excess parameter represents the number of excess neutrons per nucleon and can only change as a result of weak interactions. For example, if only ${ }^{4} \mathrm{He},{ }^{12} \mathrm{C}$, and ${ }^{16} \mathrm{O}$ are present in the plasma, then $\eta=0$.

A number of interesting properties can be derived from Eq. (4.1). First, it can be shown that, if we keep the density constant and raise the temperature, an increasing fraction of the composition resides in the light species (protons, neutrons, and $\alpha$-particles). Second, it turns out that the neutron excess parameter influences sensitively the composition during nuclear statistical equilibrium. In fact, NSE favors the abundance of that particular nuclide for which (i) the individual neutron excess is equal to the total neutron excess, and (ii) the binding energy is at maximum. For example, when $\eta \approx 0$, then ${ }^{56} \mathrm{Ni}$ (with 28 neutrons and 28 protons; $\eta_{i}=0$ ) is the most abundant species. For $\eta \approx 0.04$, the most abundant species becomes ${ }^{54} \mathrm{Fe}$ (with 28 neutrons and 26 protons; $\eta_{i}=0.037$ ), and so on. The neutron excess must be monitored very carefully during all burning stages that precede NSE. Therefore, stellar weak interaction rates must be known reliably.

\section{Direct Laboratory Measurements}

Direct measurements of reactions at the low energies of interest require high beam currents, efficient and low-background detection methods, and long running times. This is because typical count rates are low enough that the desired signals are often indistinguishable from environmental backgrounds. A useful figure of merit for a measurement is the net signal divided by the uncertainty 
in the total number of detected counts. Usually the background is sampled over a region near the signal peak and if that region is much wider than the width of the peak, then the overall uncertainty will be governed by the total number of counts in the peak (signal + background) and not by the estimate of the background to be subtracted off. In practice, if the background can be measured over an interval that is about 10 times the width of the peak, then the background estimation is precise enough not to impact the overall uncertainty. In this case, the figure of merit is

$$
\text { F.O.M. }=\frac{\mathrm{S}}{\sqrt{\mathrm{S}+\mathrm{B}}} \approx \frac{\mathrm{S}}{\sqrt{\mathrm{B}}} \text { if } \mathrm{S} \ll \mathrm{B},
$$

where $\mathrm{S}$ is the signal and B is the background. In other words, even though it is important to reduce backgrounds, it is more important to increase the signal rate, within practical limits.

Environmental backgrounds can arise from natural radioactivity such as ${ }^{40} \mathrm{~K},{ }^{208} \mathrm{Tl},{ }^{238} \mathrm{U}$ and its decay products, neutrons from natural fission and $(\alpha, \mathrm{n})$ reactions, and from radon gas. Gammarays from these sources occur at energies below $\approx 3 \mathrm{MeV}$ and can be reduced by passive shielding and by careful selection of materials used for detectors. In contrast, cosmic-ray backgrounds arising from muon-induced interactions such as direct ionization, pair-production, bremsstrahlung and nuclear interactions can be much more problematic because they occur at all energies and are not significantly attenuated in passive shields. In fact, the passive shielding itself can become a target for muon interactions and thus a source of background. However, the muon flux can be drastically reduced by going deep underground. Nonetheless, beam-induced background can often be the limiting factor in the success of a measurement, which places a premium on target purity. Significant reductions in backgrounds can also be achieved by using an array of detectors in various conicidence/anticoincidence modes, as we will discussed below.

\subsection{Accelerators}

The key reactions that influence the lives of stars place demands on accelerator performance that are difficult to meet with a single design approach. Instead, accelerators are designed more or less to address specific burning regimes. However, in all cases, run times can be measured in weeks or months and so stable operation over extended periods is critical. In addition, the energy resolution of the beam should be $\leq 1 \mathrm{keV}$ in order to permit accurate measurements of resonance energies. These requirements can be met with electrostatic accelerators, whose energies and beam species can be tailored to the needs of a particular experiment. For example, quiescent $\mathrm{H}$ burning occurs at the lowest temperatures and so beam energies of several hundreds of $\mathrm{keV}$ will usually suffice. However, beam currents should ideally be on the mA level and limited only by target degradation. The approach taken at LENA (Fig. 10) is to place a high-intensity ECR ion source on a 200-kV table [23]. Recent improvements to the source have increased the beam current on target to $2 \mathrm{~mA}$ and further upgrades are in progress with the goal of increasing the beam current to $20 \mathrm{~mA}$. Target stability is a concern with currents in this range, but the aim here is to pulse the beam with a $\sim 10 \%$ duty cycle, resulting in an average beam current of 1-2 mA on target, but at the same time, external backgrounds, which do not scale with the beam current, will be effectively reduced by a factor of 10 . The LUNA II accelerator consists of an RF-discharge ion source placed within a 400-kV Cockcroft-Walton charging structure [24]. Typical beam currents are on the order 


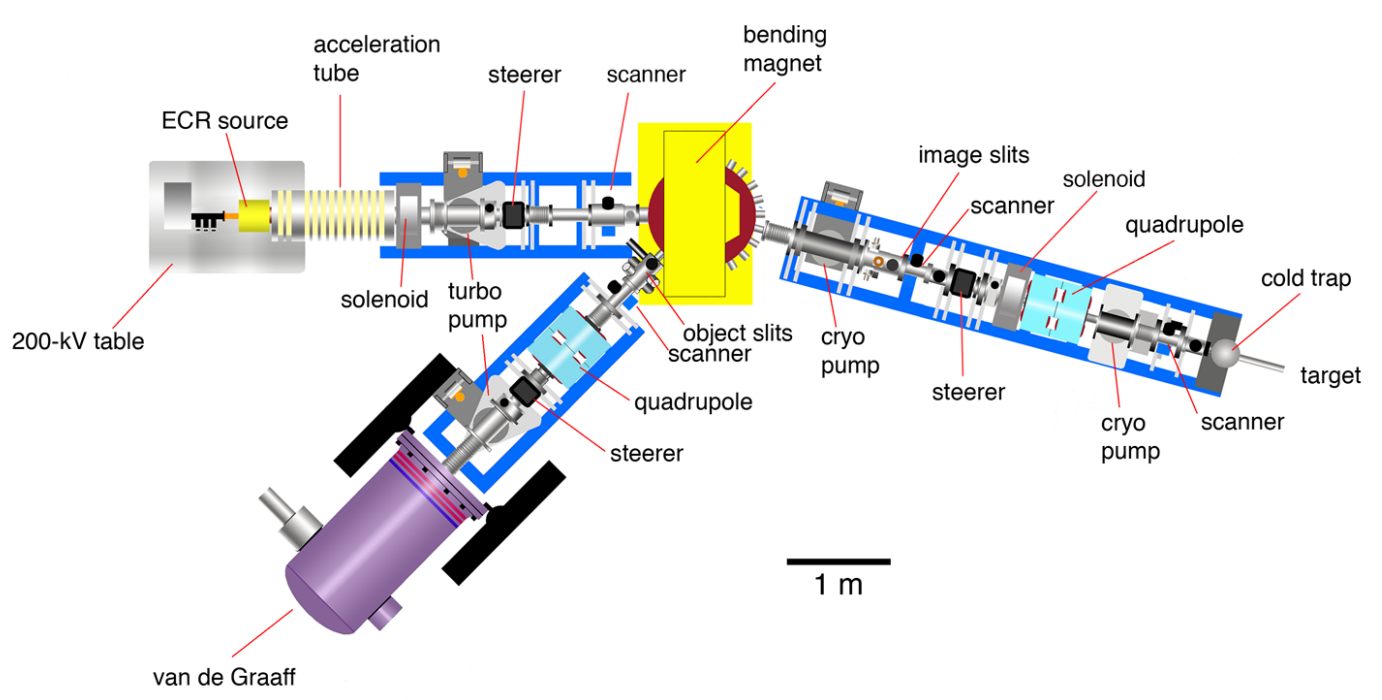

Figure 10: Schematic view of LENA.

of $0.25 \mathrm{~mA}$, but this accelerator is placed deep underground, which effectively eliminates cosmicray induced backgrounds. However, it should be noted that even in an underground environment, beam-induced and environmental backgrounds are still problematic and require careful material selection as well as passive shielding.

Measurements of more advanced burning stages, e.g., He and $\mathrm{C}$ burning, require beams with energies on the order of $0.5-5.0 \mathrm{MeV}$. Although the upper end of this energy range is beyond the stellar energies of interest, precise measurements at higher energies are often more valuable in defining features of the reaction mechanism, such as interference effects, than measurements at lower energies that could be compromised by limited statistics. In the same manner, measurements of elastic scattering in the $\mathrm{MeV}$ range can be used to probe the amplitudes of interfering resonances and provide constraints on R-matrix fits to low-energy capture data [25, 26, 27, 28]. Finally, heavyion beams in the $\mathrm{MeV}$ energy range can be used to measure $(\mathrm{p}, \gamma)$ and $(\alpha, \gamma)$ reactions in inverse kinematics. The advantage of this approach is that kinematic focusing allows the total cross section to be measured with very high efficiency, as has been demonstrated for the ${ }^{12} \mathrm{C}(\alpha, \gamma){ }^{16} \mathrm{O}$ reaction [29]. An example of an accelerator built specifically for measurements of this type is the 5-MV St. ANA machine that was recently installed at the University of Notre Dame.

\subsection{Targets}

Several considerations guide the selection of a suitable target for an experiment. A useful target must have a high concentration of the isotope of interest and must be able to withstand high beam currents for extended periods. However, cleanliness is also critically important because contaminants can give rise to significant beam-induced backgrounds that can be the limiting factor in experimental sensitivity. Solid targets are more prone to contamination than gas targets, but not every target material can be prepared in a gaseous state and gas targets present their own challenges. Typically, solid targets consist of a high concentration of the isotope of interest implanted into the first few nanometers of a nickel or tantalum backing or deposited (e.g., by evaporation, sputtering, 


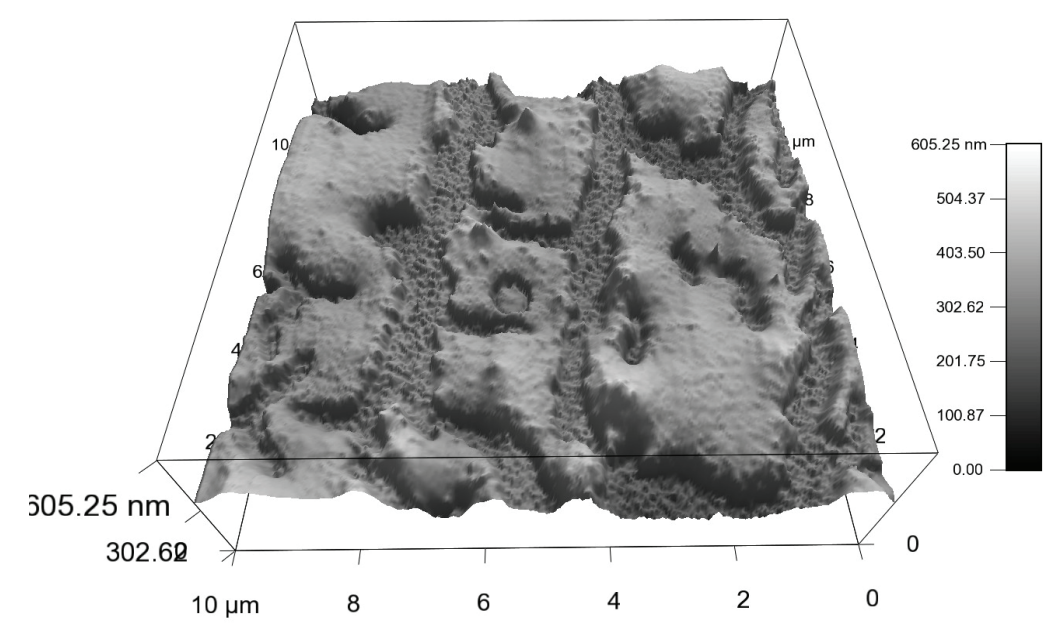

Figure 11: Atomic force microscope image of a $\mathrm{Na}_{2} \mathrm{WO}_{4}$ target after bombardment by protons. The valleys correspond to locations where the evaporated $\mathrm{Na}_{2} \mathrm{WO}_{4}$ (the raised areas) has been removed down to the tantalum target backing. The mechanism responsible for the non-uniform degradation is presently not understood.

or anodization) onto a substrate. Surface contaminants can be introduced during the manufacturing of the backings, as well as when they are machined to size. Such contaminants can be reduced by placing the backing in an acetone bath and by wet etching the backing in acid. Outgassing the backing by resistive heating while under vacuum will also drive out contaminants near the surface. For instance, acid etching is very effective at removing carbon contamination, while outgassing significantly reduces ${ }^{19} \mathrm{~F}$. Storage can also introduce contaminants and in our experience storing the targets in compressed nitrogen gas ( $2 \mathrm{~atm}$ ) is preferable to storing them in vacuum.

There are a number of challenges encountered when using solid targets. For example, they will inevitably degrade because of beam heating or implantation of beam particles (Fig. 11), necessitating the preparation of a number of targets for a single measurement. In addition, there is a limit to the cleanliness of targets prepared by surface deposition because the material used will inevitably contain trace quantities of other substances. Also, in most instances solid targets are compounds or amalgamations of the isotope of interest with other elements. This lowers the density of the target isotope and reduces the reaction yield. Finding the best fabrication procedure and backing to produce clean targets that are able to withstand high beam currents can be a lengthy development process.

In contrast with solid targets, gas targets can be very pure and a windowless target will not be degraded by the beam. However, beam heating can still be a problem because of resulting changes in gas density. This is more of an issue with extended targets than with gas jets. Gas jets, while far more complicated to build, have the advantage of a smaller gas volume and, therefore, can withstand higher beam currents. The resulting increase in reaction yield is partially offset by the fact that the nozzles (used to produce the jet) and accompanying pumping apertures require the detectors to be placed farther from the interaction region compared to solid or extended gas targets. These elements can also produce background if struck by the beam. In summary, there is no single 
ideal approach to targets and the choice of target is usually dictated by the specific reaction to be measured.

\subsection{Detectors}

Every reaction of interest represents a special case from the standpoint of detection technique. The detection of charged particles versus neutrons versus $\gamma$-rays implies very different detection philosophies, but even if we restrict our attention to $(\mathrm{p}, \gamma)$ reactions, there is no single detector geometry that will be ideal for every measurement. Therefore, it is important to have a flexible detector setup and, in all cases, high detection efficiency and high resolving power are required.

Large-volume HPGe detectors are usually preferred for $\gamma$-ray detection on the basis of their high resolution. Assuming that the residual background is uniformly distributed in energy, high resolution minimizes the amount of background that is sampled over the region of the signal peak, thus maximizing the signal-to-background ratio. In addition, HPGe crystals have a very low internal activity, and the technology for low background cryostats and pre-amplifiers is well developed and commercially available. Finally, even a large-volume detector is still small enough to be efficiently shielded and there are well-developed techniques for passive shielding. The disadvantage of a HPGe detector is the relatively low full-energy peak efficiency as compared to scintillation counters. However, the comparatively poor energy resolution of scintillation detectors limits their usefulness in low signal-to-background applications. Nonetheless, scintillation detectors can be used as calorimeters, where nearly the total decay energy is collected with efficiencies of about 50$70 \%$. The signal detected is the summed $\gamma$-ray energy corresponding to the characteristic Q-value of the reaction. An example of this approach is the $4 \pi$ BGO detector used at LUNA [30].

If the signal of interest is associated with a $\gamma \gamma$ cascade, then there are a number of different coincidence conditions that can be selected in order to optimize detection sensitivity. For example, energy and multiplicity cuts can be used to reduce backgrounds, particularly those associated with environmental sources. This is accomplished at LENA by using a HPGe detector surrounded by a 16-element $\mathrm{NaI}(\mathrm{Tl})$ annulus [31] (Fig. 12). Energy cuts are applied as

$$
E_{\text {min }}<E_{G e}+E_{N a I}<E_{\text {max }}
$$

where $E_{\min } \approx 3 \mathrm{MeV}$ is usually sufficient to reduce room background and $E_{\max }$ is usually chosen to be slightly larger than the excitation energy of the populated levels in the reaction of interest, eliminating events with total energies above the decay energy. In this approach, the continuum background for $\mathrm{E}_{G e}<3 \mathrm{MeV}$ is reduced by a factor of $\sim 100$ beyond what is obtained with passive and active shielding, which permits low-energy in-beam $\gamma$-rays to be observed. Since all Compton events that occur within the energy gate in the $\mathrm{NaI}(\mathrm{Tl})$ annulus are also accepted, the HPGe coincidence efficiency can be approximately $60 \%$ of the singles efficiency.

The neutrons produced in different $(\alpha, \mathrm{n})$ reactions in stars have energies on the order of $<$ $1 \mathrm{MeV}\left(<2.5 \mathrm{MeV}\right.$ for $\left.{ }^{13} \mathrm{C}(\alpha, n){ }^{16} \mathrm{O}\right)$. These are typically measured using a (nearly) $4 \pi$ detector consisting of ${ }^{3} \mathrm{He}$ counters embedded in a polyethylene moderator matrix. These detectors have a high counting efficiency of around 50\% for low energy neutrons created at the center of the detector. Examples are the detector used to measure the ${ }^{22} \mathrm{Ne}(\alpha, \mathrm{n})^{25} \mathrm{Mg}$ reaction at Stuttgart [32], and the NERO detector at MSU [33]. It should be noted that these detectors simply count neutrons 


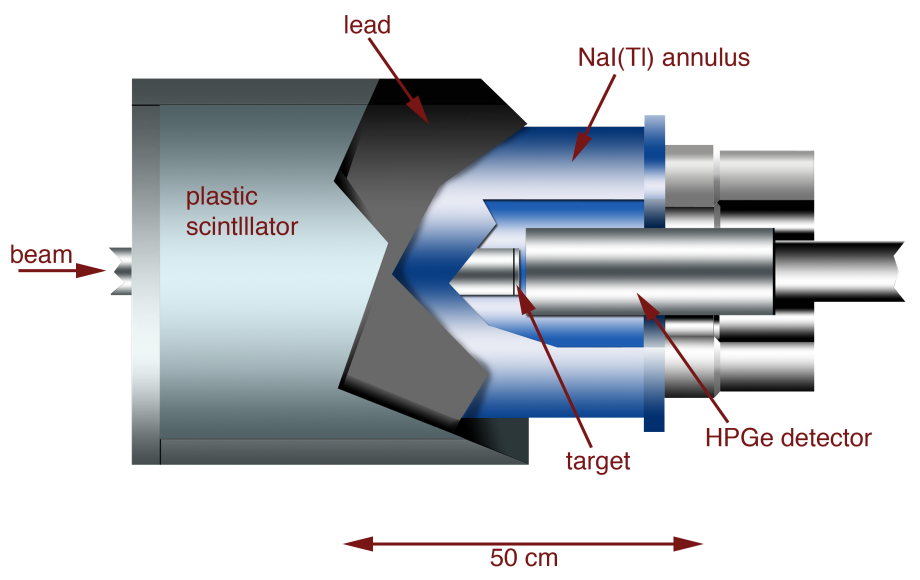

Figure 12: The $\gamma$-ray coincidence spectrometer used at LENA. It consists of a $135 \%$ coaxial HPGe detector surrounded by a 16-element annulus of $\mathrm{NaI}(\mathrm{Tl}), 33.0 \mathrm{~cm}$ in length, with outer and inner diameters of 35.7 $\mathrm{cm}$ and $11.8 \mathrm{~cm}$, respectively. The plastic scintillators form a muon veto.

and provide no energy information. This places a premium on reducing neutron backgrounds, particularly beam-induced $(\alpha, \mathrm{n})$ reactions.

The charged-particle reactions of astrophysical interest are typically $(\mathrm{p}, \alpha)$ reactions. The standard detection approach is to place $\mathrm{Si}(\mathrm{SB})$ detectors in close geometry with respect to the target. Thin, metallic foils are placed over the detectors to reduce the intense flux of scattered protons that would quickly destroy an unshielded detector. While this setup is simple in concept, it is challenging to achieve in practice. That is because a foil thick enough to stop a proton with an energy of a few hundred $\mathrm{keV}$ will also significantly reduce the energies of the $\alpha$-particles, which are on the order of 1-2 MeV. Straggling will also significantly degrade the energy resolution of the detected $\alpha$-particles. In addition, foils are not perfectly uniform, giving rise to an additional broadening of the expected peak. The net result is that the $\alpha$-particles of interest are difficult to distinguish from low-energy background and noise. Background from pileup can be reduced by replacing the $\mathrm{Si}(\mathrm{SB})$ detectors with Si strip detectors. However, these must still be shielded from scattered beam.

\section{Outlook}

We have now reached the end of this brief survey of nuclear astrophysics. A recent review [22] of the field identified 15 key questions: (i) Why do predictions of helioseismology disagree with those of the standard solar model? (ii) What is the solution to the lithium problem in Big Bang nucleosynthesis? (iii) What do the observed light-nuclide and s-process abundances tell us about convection and dredge-up in massive stars and AGB stars? (iv) What are the production sites of the $\gamma$-ray emitting radioisotopes ${ }^{26} \mathrm{Al},{ }^{44} \mathrm{Ti}$ and ${ }^{60} \mathrm{Fe}$ ? (v) What is the origin of about 30 rare and neutron-deficient nuclides beyond the iron peak (p-nuclides)? (vi) What causes core-collapse supernovae to explode? (vii) What is the extent of neutrino-induced nucleosynthesis ( $v$-process)? (viii) What is the extent of the nucleosynthesis in proton-rich outflows in the early ejecta of core- 
collapse supernovae ( $v$ p-process)? (ix) What are the sites of the r-process? (x) What causes the discrepancy between models and observations regarding the mass ejected during classical nova outbursts? (xi) Which are the physical mechanisms driving convective mixing in novae? (xii) What are the progenitors of type Ia supernovae? (xiii) What is the nucleosynthesis endpoint in type I x-ray bursts? Is there any matter ejected from those systems? (xiv) What is the impact of stellar mergers on Galactic chemical abundances? (xv) What are the production and acceleration sites of Galactic cosmic rays? The student may note with interest the many areas of nuclear physics that are sampled by stars. It is gratifying to see that, after many decades of research, we are in possession of a remarkably successful theory of stellar evolution and nucleosynthesis. It is equally exciting that there are still many unsolved questions in nuclear astrophysics, some of which will hold important implications for related fields, such as cosmology, meteoritics, and cosmochronology. Thus the future looks bright for aspiring young minds!

\section{References}

[1] H. A. Bethe, and C. L. Critchfield, Phys. Rev. 54, 248 (1938).

[2] H. A. Bethe, Phys. Rev. 55, 434 (1939).

[3] C. F. von Weizsäcker, Phys. Z. 39, 633 (1938).

[4] E. M. Burbidge, G. R. Burbidge, W. A. Fowler, and F. Hoyle, Rev. Mod. Phys. 29, 547 (1957).

[5] A. G. W. Cameron, Pub. Astron. Soc. Pac. 69, 201 (1957).

[6] K. Lodders, Astrophys. J. 591, 1220 (2003).

[7] C. Iliadis, Nuclear Physics of Stars, 2nd (revised and enlarged) Edition, Wiley-VCH, Weinheim (2015).

[8] N. Prantzos, C. Charbonnel and C. Iliadis, Astron. Astrophys. 470, 179 (2007).

[9] C. Iliadis, Nucl. Phys. 618, 166 (1997).

[10] D. Arnett, Supernovae and Nucleosynthesis, Princeton University Press, Princeton (1996).

[11] G. R. Caughlan, and W. A. Fowler, At. Data Nucl. Data Tab. 40, 284 (1988).

[12] C. Angulo, et al., Nucl. Phys. A 656, 3 (1999).

[13] C. Iliadis, et al., Astrophys. J. Suppl. 134, 151 (2001).

[14] R. Longland et al., Nucl. Phys. A 841, 1 (2010).

[15] C. Iliadis et al., Nucl. Phys. A 841, 31 (2010).

[16] C. Iliadis et al., Nucl. Phys. A 841, 251 (2010).

[17] C. Iliadis et al., Nucl. Phys. A 841, 323 (2010).

[18] J. N. Bahcall, and R. M. May, Astrophys. J. 155, 511 (1969).

[19] H. Costantini, et al., Rep. Prog. Phys. 72, 086301 (2009).

[20] R. Runkle, et al., Phys. Rev. Lett. 94, 082503 (2005).

[21] F. Hoyle, Astrophys. J. Suppl. 1, 121 (1954).

[22] J. José and C. Iliadis, Rep. Prog. Phys. 74, 096901 (2011). 
[23] J. M. Cesaratto, et al., Nucl. Instrum. Meth. A 623, 888 (2010).

[24] A. Formicola et al., Nucl. Instrum. Meth. A 507, 609 (2003).

[25] M. D’Agostino Bruno, et al., Nuovo Cimento Ser. A 27, 1 (1975).

[26] R. Plaga, et al., Nucl. Phys. A 465, 291 (1987).

[27] P. Tischhauser, et al., Phys. Rev. C 79, 055803 (2009).

[28] R.J. DeBoer, et al., Phys. Rev. C, submitted (2015).

[29] D. Schürmann, et al. Phys. Lett. B 711, 35 (2012).

[30] C. Casella et al., Nucl. Instrum. Meth. A 489, 160 (2002).

[31] R. Longland et al., Nucl. Instrum. Meth. A 566, 452 (2006).

[32] M. Jaeger et al., Phys. Rev. Lett. 87, 202501 (2001).

[33] J. Pereira et al., Nucl. Instrum. Meth. A 618, 275 (2010). 